\title{
A Novel Adaptive Turbo Receiver for Large-Scale MI MO Communications
}

\author{
Yu-Kuan Chang ${ }^{1}$, Fang-Biau Ueng ${ }^{1}$, and Bo-Yi Tsai ${ }^{1}$ \\ ${ }^{1}$ Department of Electrical Engineering \\ National Chung Hsing University \\ Taichung, Taiwan \\ Email: fbueng@nchu.edu.tw \\ *Corresponding author: Fang-Biau Ueng
}

Received November 7, 2017; revised January 8, 2017; accepted January 30, 2018;

Published July 31, 2018

\begin{abstract}
Massive (large-scale) MIMO (multiple-input multiple-output) is one of the key technologies in next-generation wireless communication systems. This paper proposes a high-performance low-complexity turbo receiver for SC-FDMA (single-carrier frequency-division multiple access) based MMIMO (massive MIMO) systems. Because SC-FDMA technology has the desirable characteristics of OFDMA (orthogonal frequency division multiple access) and the low PAPR (peak-to-average power ratio) of SC transmission schemes, the 3GPP LTE (long-term evolution) has adopted it as the uplink transmission to meet the demand high data rate and low error rate performance. The complexity of computing will be increased greatly in base station with massive MIMO (MMIMO) system. In this paper, a low-complexity adaptive turbo equalization receiver based on normalized minimal symbol-error-rate for MMIMO SC-FDMA system is proposed. The proposed receiver is with low complexity than that of the conventional turbo MMSE (minimum mean square error) equalizer and is also with better bit error rate (BER) performance than that of the conventional adaptive turbo MMSE equalizer. Simulation results confirm the effectiveness of the proposed scheme.
\end{abstract}

Keywords: Multiple Input Multiple Output, SC-FDMA, Minimal Symbol-Error-Rate, Turbo Equalization 


\section{Introduction}

In the Long Term Evolution Advanced (LTE-A), the uplink employs SC-FDMA [1] and the downlink employs OFDMA [2]. SC-FDMA is a modified version of the OFDM. MIMO wireless communication which uses spatial multiplexing has the capability to increase capacity through added data, which needs to be separated by a detection algorithm. With reduced complexity, the performance of SDs (sphere decoders) approaches to that of the maximum likelihood (ML) detector. However, SDs still have a high complexity [3]. The low-complexity SISO (soft-input soft-output) equalization that is based on MMSE criterion has been developed [4]. It includes a new approach which has lower complexity than optimal MMSE solution and the extension of the existing approach to a general signal constellation [5]. Existing transmit antenna diversity techniques such as STBC (space time block coding) [6] and SFBC (space frequency block coding) [7] are incompatible either with the system constraints or with the single-carrier nature of SC-FDMA. However, these techniques need to compute channel parameter. The spatial multiplexing technique [8] and the adaptive equalizer can be chosen to prevent the estimation of the channel, and the complexity can be reduced. Traditionally, adaptive algorithms have been developed based on the MMSE or the least mean square (LMS) criterion [9]. The MMSE solution is not optimal in equalization application. Some researches those are based on the minimum bit error rate (MBER) criterion or the minimum symbol error rate (MSER) criterion have been conducted to find alternative designs $[10,11]$. Some other researches which are based on MSER include channel equalization [11-15], beamforming [16], carrier phase recovery [17], timing recovery [18], precoding [19] and power control/allocation [20-22]. Compared to the existing works of MSER/MBER equalizers [11][12], the constrained optimization problem of the equalizer is solved with the Lagrange multiplier method [22, 23].

To increase its practicability, this paper proposes a low-complexity normalized adaptive MSER (AMSER) equalizer for MMIMO SC-FDMA system. To reduce the error propagation and enhance the performance, the turbo code and the optimal equalizer are combined as the turbo equalization [24]. The iterative property of turbo receiver is employed to improve the accuracy of estimated data. The proposed scheme is called as normalized adaptive minimal symbol-error-rate (NAMSER) turbo equalizer. Compared with some existing works which are LMS turbo equalizer [25], MMSE turbo equalizer [26] and AMSER equalizer [12] with turbo receiver, the proposed scheme has better performance and lower complexity. The simulation results show that the proposed scheme has better error rate performance than that of the existing schemes. Considering hardware implementation, the look-up table approximation [27, 28] can be employed to approximate hyperbolic tangent.

In the future, MMIMO technique is one of the important parts in 5G communication [29, 30]. In [30], it discusses the complexity of base station (BS) detection which is used in LTE uplink system. The complexity of computing will be increased greatly in BS with large-scale MIMO system as opposed the small-scale MIMO system which is 2-to-4 antennas at transmitter and receiver. In uplink system, most critical mission is to reduce complexity of data detection and power consumption. As the hundreds of antennas at the BS, we have a large dimension of channel matrix. For MMIMO system, the optimum detections such as the ML detection or the SD detection have very high computational complexity. For some sub-optimal algorithm like MMSE, the matrix multiplication and the inverse matrix should be computed. So the complexity will be an exponential growth along with amount of antennas at BS. In [31, 
32], they use ZF (zero-forcing) and MMSE detectors in the receiver. But they still have high complexity with computation of channel inverse matrix. In [33] and [34], they use MMSE algorithm in massive MIMO system and use their method to reduce complexity. But it still has to compute inverse matrix of large dimensional channel and the number of antenna is not large enough. In [35][36], one shows that the MRC (maximum ratio combining) achieves the same throughput as that of ZF for large number of antennas. Some optimal algorithm, such as ML in [37] and SD [38][39], they investigate maximum likelihood. For heuristic detectors, these optimal algorithms have better performance, however the number of antenna still not large enough, and they easily reach the high complexity. Therefore, low-complexity linear detection algorithms that with sub-optimal error rate performance and scale favorably to the high-dimensional problems is important for MMIMO system. The proposed scheme employs a transform matrix which combines the received data to solve the high dimensional channel matrix. The system can be seen as a linear growth in MMIMO. In other words, this paper finds a possibility of low-complexity data detection in MMIMO systems. The organization of this paper is as follows. In section 2, the proposed system model is described. In section 3 , the proposed normalized adaptive equalization turbo receiver are described in detail. Simulation results and conclusions are provided in section 4 and section 5, respectively.

\section{System Model}

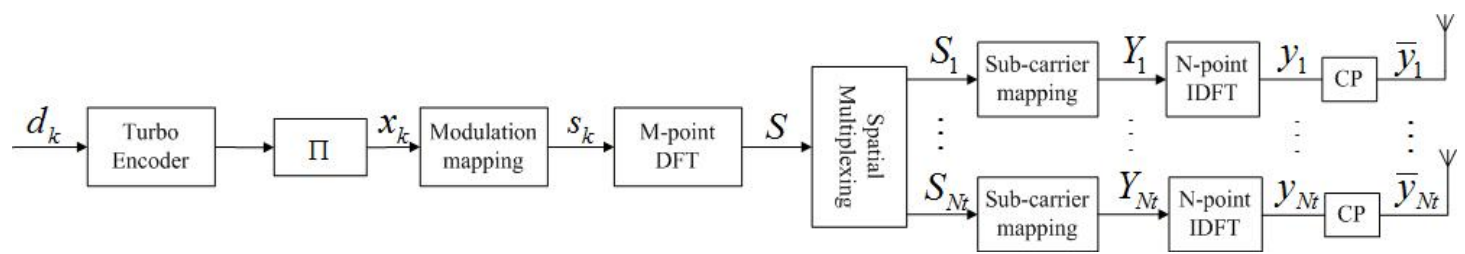

Fig. 1. The transmitter of the proposed MMIMO SC-FDMA systems.

In this section, a MMIMO SC-FDMA system is considered, as illustrated in Fig. 1. Each original symbol $d_{k}$ is encoded to coded symbol $x_{k}$. The $s_{k}$ is a BPSK/QPSK signal within the $k$ th symbol time. After M-point discrete Fourier transform (DFT), the signal can be written as

$$
\mathrm{S}=\mathbf{F}_{M} S
$$

Where $\mathbf{F}_{\mathbf{M}}$ is the $M$-point discrete Fourier transform matrix. The element of the matrix can be written as

$$
\left[\mathbf{F}_{M}\right]_{a, b}=\frac{1}{\sqrt{M}} \mathrm{e}^{\left(\frac{-2 \pi a b}{M}\right)} \quad a, b \in\{0,1, \ldots, M-1\}
$$

Next we will do spatial multiplexing (SM), we assume two transmitted antennas to explain how SM works. The signal must be separated into two components, even frequency signal and odd frequency signal, it can be shown as following,

$$
\mathbf{S}=\left\{\begin{array}{c}
\mathbf{S}_{2 i} \\
\mathbf{S}_{2 i+1}
\end{array},\right.
$$

the first antenna transmits $\mathbf{S}_{2 i}$, the second antenna transmits $\mathbf{S}_{2 i+1}$. The signal becomes $y_{N_{t}}$ and then we add CP (cyclic prefix). The signal becomes $\overline{\mathbf{y}}_{N_{t}}$ and then transmitted. Through the fading channel, we collect all $N_{t}$ antennas into a $\left(N+L_{c p}\right) \times N_{t}$ matrix.

$$
\overline{\mathbf{y}}_{N_{t}}=\left[\bar{y}_{N_{t}}(0), \bar{y}_{N_{t}}(1), \ldots, \bar{y}_{N_{t}}\left(N+L_{c p}-1\right)\right]
$$


That means the signal of (4) becomes $\overline{\mathbf{y}}=\left[\begin{array}{llll}\overline{\mathrm{y}}_{1} & \overline{\mathrm{y}}_{2} & \ldots & \overline{\mathrm{y}}_{N_{t}}\end{array}\right]$. The signal at the $n_{\mathrm{r}}$ th antenna is denoted as $\mathbf{z}_{N_{r}}$,

$$
\mathbf{z}_{N_{r}}=\widetilde{\mathbf{h}}_{N_{r}, N_{t}} \otimes \overline{\mathbf{y}}+\mathbf{n}_{N_{r}}
$$

Where $\tilde{\mathbf{h}}_{N_{r}, N_{t}}$ is a $\left(N_{r} L\right) \times N_{t}$ channel matrix shown as follows,

$$
\tilde{\mathbf{h}}_{N_{r}, N_{t}}=\left[\begin{array}{cccc}
h_{1,1} & h_{1,2} & \ldots & h_{1, N_{t}} \\
h_{2,1} & h_{2,2} & \cdots & h_{2, N_{t}} \\
\vdots & \vdots & \ddots & \vdots \\
h_{N_{r}, 1} & h_{N_{r}, 2} & \cdots & h_{N_{r}, N_{t}}
\end{array}\right]
$$

The element vector of channel matrix is described as follows,

$$
\mathbf{h}_{N_{r}, N_{t}}=\left[\begin{array}{llll}
h_{N_{r}, N_{t}}(0) & h_{N_{r}, N_{t}}(1) & \cdots & h_{N_{r}, N_{t}}(L-1)
\end{array}\right]^{T}
$$

where (5) means the transmitted signal $\overline{\mathbf{y}}$ do convolution with channel $\widetilde{\mathbf{h}}_{N_{r}, N_{t}}$, and $\mathbf{n}_{N_{r}}$ is complex additive white Gaussian noise. After $N$-point DFT, the signal becomes

$$
\overline{\mathbf{R}}_{N_{r}}=\mathbf{F}_{N}\left(\tilde{\mathbf{h}}_{N_{r}, N_{t}} \otimes \overline{\mathbf{y}}+n_{N_{r}}\right)=\overline{\mathbf{H}}_{N_{r}, N_{t}} \mathbf{Y}_{N_{t}}+\Lambda_{N_{r}}
$$

where $\overline{\mathbf{H}}_{N_{r}, N_{t}}=\mathbf{F}_{N} \otimes \tilde{\mathbf{h}}_{N_{r}, N_{t}}, \boldsymbol{\Lambda}_{N_{r}}=\mathbf{F}_{N} n_{N_{r}}, \mathbf{F}_{N}$ is $N$-point DFT matrix. The received symbol $\overline{\mathbf{R}}_{k}$ are used to perform equalization in first iteration. The architecture of the proposed turbo NAMSER scheme is shown in Fig. 2. The output of decoder is the estimated value of transmitted bits $d_{k}$, and decoder will calculate LLR value which will be used to compute a new estimation $s_{k}^{d}$ of $s_{k}$, then it will be used in next iteration to produce a more accurate estimation of $s_{k}$. We use received symbol and extrinsic information of turbo decoder in the proposed NAMSER turbo equalizer.

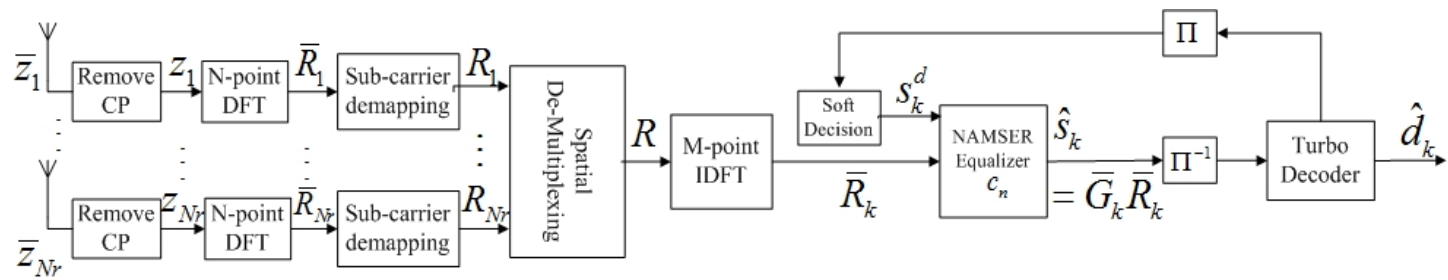

Fig. 2. The proposed receiver for the MMIMO SC-FDMA systems.

\section{The Proposed NAMSER Turbo Equalizer}

\subsection{NAMSER Equalization}

At time instant $k$, the equalizer at the previous stage, denoted as $\overline{\mathbf{G}}_{k-1}$, is available. $\overline{\mathbf{G}}_{k}$ is updated based on $\overline{\mathbf{G}}_{k-1}$ and the new received signals $\overline{\mathbf{R}}_{k}$. The constraint is that $\overline{\mathbf{G}}_{k}$ will provide correct symbols detection which is $\hat{\mathbf{s}}_{k}=\overline{\mathbf{G}}_{k}^{T} \overline{\mathbf{R}}_{k}$. The objective function is

$$
\min _{\overline{\mathbf{G}}_{k}}\left\|\overline{\mathbf{G}}_{k}-\overline{\mathbf{G}}_{k-1}\right\|^{2}
$$

We will derive an extension of the NAMBER algorithm to the QPSK source. We still use the model of (8), but the real and imaginary parts of $s_{k}$ are independently and uniformly drawn from $\{ \pm 1\}$. At first, we consider the real part of the equalizer output,

$$
\mathfrak{R}\left\{\hat{s}_{k}\right\}=\Re\left\{\overline{\mathbf{G}}_{k}^{T} \overline{\mathbf{R}}_{k}\right\}=\overline{\mathbf{G}}_{k, R}^{T} \overline{\mathbf{R}}_{k, R}-\overline{\mathbf{G}}_{k, I}^{T} \overline{\mathbf{R}}_{k, I}=\bar{g}_{k}^{T} \bar{r}_{k}
$$


where $\overline{\mathbf{G}}_{k, R}^{T}, \overline{\mathbf{R}}_{k, R}$ and $\overline{\mathbf{G}}_{k, I}^{T}, \overline{\mathbf{R}}_{k, I}$ are the real parts and imaginary parts of the equalizer $\overline{\mathbf{G}}_{k}$ and received signal $\overline{\mathbf{R}}_{k}$. And $\bar{g}_{k}=\left[\overline{\mathbf{G}}_{k, R}^{T}-\overline{\mathbf{G}}_{k, I}^{T}\right]^{T}, \bar{r}_{k}=\left[\bar{R}_{k, R}^{T}, \bar{R}_{k, I}^{T}\right]^{T}$. We want to update $\bar{g}_{k}$ to minimize $\operatorname{Prob}\left\{\mathfrak{R}\left\{\hat{s}_{k}\right\} \neq \Re\left\{S_{k}\right\}\right\}$ without regarding to the imaginary part of the detection. The problem model is described as follows,

$$
\min _{\bar{g}_{k}}\left\|\bar{g}_{k}-\bar{g}_{k-1}\right\|^{2} \text { subject to } \mathfrak{R}\left\{\hat{s}_{k}\right\}=\mathfrak{R}\left\{s_{k}\right\}
$$

We consider the range of bias between the real symbol and the equalizer output signal, given by $-1<\bar{g}_{k}^{T} \bar{r}_{k}-\mathfrak{R}\left\{s_{k}\right\}<1$. The above derivation can be extended to other decision ranges. We rewrite the constraint of (11) as

$$
\bar{g}_{k}^{T} \bar{r}_{k}-\Re\left\{s_{k}\right\}>-1
$$

and

$$
\bar{g}_{k}^{T} \bar{r}_{k}-\mathfrak{R}\left\{s_{k}\right\}<1
$$

The constraints can be equivalently written as

$$
\operatorname{sgn}\left(\bar{g}_{k}^{T} \bar{r}_{k}-\Re\left\{s_{k}\right\}+1\right)=1
$$

and

$$
\operatorname{sgn}\left(\bar{g}_{k}^{T} \bar{r}_{k}-\Re\left\{s_{k}\right\}-1\right)=-1
$$

Combine (14) and (15) as follows,

$$
\operatorname{sgn}\left(\bar{g}_{k}^{T} \bar{r}_{k}-\Re\left\{s_{k}\right\}+1\right)+\operatorname{sgn}\left(\bar{g}_{k}^{T} \bar{r}_{k}-\mathfrak{R}\left\{s_{k}\right\}-1\right)=0
$$

To make the objective function differentiable, we approximate $\operatorname{sgn}\left(\bar{g}_{k}^{T} \bar{r}_{k}\right)$ with $\tanh \left(\omega\left(\bar{g}_{k}^{T} \bar{r}_{k}\right)\right)$ where $\omega$ is a sufficiently large number. Then the problem (11) can be rewritten as

$$
\min _{\bar{g}_{k}}\left\|\bar{g}_{k}-\bar{g}_{k-1}\right\|^{2} \text { subject to } \tanh \left(\omega\left(\bar{g}_{k}^{T} \bar{r}_{k}-\mathfrak{R}\left\{s_{k}\right\}+1\right)\right)+\tanh \left(\omega\left(\bar{g}_{k}^{T} \bar{r}_{k}-\Re\left\{s_{k}\right\}-1\right)\right)=0
$$

Define the objective function as follows,

$$
C\left(\bar{g}_{k}\right)=\left\|\bar{g}_{k}-\bar{g}_{k-1}\right\|^{2}+\lambda\left(\tanh \left(\omega\left(\bar{g}_{k}^{T} \bar{r}_{k}-\mathfrak{R}\left\{s_{k}\right\}+1\right)\right)+\tanh \left(\omega\left(\bar{g}_{k}^{T} \bar{T}_{k}-\mathfrak{R}\left\{s_{k}\right\}-1\right)\right)\right)=0
$$

where $\lambda$ is the Lagrange multiplier. Differentiating (18), we can obtain

$$
\frac{\partial \mathrm{C}\left(\bar{g}_{k}\right)}{\partial \bar{g}_{k}}=2\left(\bar{g}_{k}-\bar{g}_{k-1}\right)+\omega \lambda\left(\tanh ^{\prime}\left(\omega\left(\bar{g}_{k}^{T} \bar{r}_{k}-\mathfrak{R}\left\{s_{k}\right\}+1\right)\right)+\tanh ^{\prime}\left(\omega\left(\bar{g}_{k}^{T} \bar{r}_{k}-\mathfrak{R}\left\{s_{k}\right\}-1\right)\right)\right) \bar{r}_{k}
$$

Setting (18) to zero, we get the adaptive algorithm as follows,

$$
\bar{g}_{k}=\bar{g}_{k-1}-\frac{1}{2} \omega \lambda\left(\tanh ^{\prime}\left(\omega\left(\bar{g}_{k}^{T} \bar{r}_{k}-\Re\left\{s_{k}\right\}+1\right)\right)+\tanh ^{\prime}\left(\omega\left(\bar{g}_{k}^{T} \bar{r}_{k}-\Re\left\{s_{k}\right\}-1\right)\right)\right) \bar{r}_{k}
$$

Substituting (19) into (17), using first-order Taylor series to approximate $\tanh (x)$, and using $\Phi_{R}=\bar{g}_{k-1}^{T} \bar{r}_{k}-\mathfrak{R}\left\{s_{k}\right\}$, we obtain

$$
\begin{array}{r}
\frac{1}{2} \omega \lambda\left(\tanh ^{\prime}\left(\omega\left(\bar{g}_{k}^{T} \bar{r}_{k}-\mathfrak{R}\left\{s_{k}\right\}+1\right)\right)+\tanh ^{\prime}\left(\omega\left(\bar{g}_{k}^{T} \bar{r}_{k}-\mathfrak{R}\left\{s_{k}\right\}-1\right)\right)\right) \\
=\frac{\tanh \left(\omega\left(\Phi_{\mathrm{R}}+1\right)\right)+\tanh \left(\omega\left(\Phi_{\mathrm{R}}-1\right)\right)}{\omega\left(\tanh ^{\prime}\left(\omega\left(\Phi_{\mathrm{R}}+1\right)\right)+\tanh ^{\prime}\left(\omega\left(\Phi_{\mathrm{R}}-1\right)\right)\right) \bar{r}_{k}^{T} \bar{r}_{k}}
\end{array}
$$

We then have $\Phi_{\mathrm{R}} \approx 0$ and consequently $\omega \tanh ^{\prime}\left(\omega\left(\Phi_{R} \pm 1\right)\right) \approx \omega \tanh ^{\prime}(\omega)$ is a constant. 
Substituting (21) into (20), we have

$$
\bar{g}_{k}=\bar{g}_{k-1}-\frac{\mu \bar{r}_{k}}{\bar{r}_{r}^{T} \bar{r}_{k}}\left(\tanh \left(\omega\left(\Phi_{R}+1\right)\right)+\tanh \left(\omega\left(\Phi_{R}-1\right)\right)\right)
$$

For the imaginary part of the equalizer output signal, we have

$$
\mathfrak{\Im}\left\{s_{k}\right\}=\mathfrak{\Im}\left\{\bar{G}_{k}^{T} \bar{R}_{k}\right\}=\bar{G}_{k, R}^{T} \bar{R}_{k, I}+\bar{G}_{k, I}^{T} \bar{R}_{k, R}=\bar{g}_{k}^{T} \mathbf{P} \bar{r}_{k}
$$

where $\mathbf{P}$ is a permutation matrix given by

$$
\mathbf{P}=\left[\begin{array}{cc}
0 & \mathbf{I} \\
-\mathbf{I} & 0
\end{array}\right]
$$

Using $\Phi_{I}=\bar{g}_{k-1}^{T} \mathbf{P} \bar{r}_{k}-\mathfrak{J}\left\{s_{k}\right\}$, it is straightforward to obtain the following update algorithm,

$$
\bar{g}_{k}=\bar{g}_{k-1}-\frac{\mu \mathbf{P} \bar{r}_{k}}{\bar{r}_{k}^{T} \bar{r}_{k}}\left(\tanh \left(\omega\left(\Phi_{I}+1\right)\right)+\tanh \left(\omega\left(\Phi_{I}-1\right)\right)\right)
$$

We rewrite (22) in vector type

$$
\left[\begin{array}{c}
\bar{G}_{k, R}^{T} \\
-\bar{G}_{k, I}^{T}
\end{array}\right]=\left[\begin{array}{c}
\bar{G}_{k-1, R}^{T} \\
-\bar{G}_{k-1, R}^{T}
\end{array}\right]-\frac{\mu}{\bar{r}_{k}^{T} \bar{r}_{k}}\left(\tanh \left(\omega\left(\Phi_{R}+1\right)\right)+\tanh \left(\omega\left(\Phi_{R}-1\right)\right)\right)\left[\begin{array}{c}
\bar{R}_{k, R}^{T} \\
\bar{R}_{k, I}^{T}
\end{array}\right]
$$

We rewrite (25) in vector type

$$
\begin{gathered}
{\left[\begin{array}{c}
\bar{G}_{k, R}^{T} \\
-\bar{G}_{k, I}^{T}
\end{array}\right]=\left[\begin{array}{c}
\bar{G}_{k-1, R}^{T} \\
-\bar{G}_{k-1, R}^{T}
\end{array}\right]-\frac{\mu}{\bar{r}_{k}^{T} \bar{r}_{k}}\left(\tanh \left(\omega\left(\left(\Phi_{I}+1\right)\right)\right)+\tanh \left(\omega\left(\Phi_{I}-1\right)\right)\right)\left[\begin{array}{cc}
0 & \mathbf{I} \\
-\mathbf{I} & 0
\end{array}\right]\left[\begin{array}{c}
\bar{R}_{k, R}^{T} \\
\bar{R}_{k, I}^{T}
\end{array}\right]} \\
=\left[\begin{array}{c}
\bar{G}_{k-1, R}^{T} \\
-\bar{G}_{k-1, R}^{T}
\end{array}\right]-\frac{\mu}{\bar{r}_{k}^{T} \bar{r}_{k}}\left(\tanh \left(\omega\left(\Phi_{I}+1\right)\right)+\tanh \left(\omega\left(\Phi_{I}-1\right)\right)\right)\left[\begin{array}{c}
\bar{R}_{k, I}^{T} \\
-\bar{R}_{k, R}^{T}
\end{array}\right]
\end{gathered}
$$

Now we let (26) and (27) times a vector $[1,-j]$ and obtain (28) and (29)

$$
\begin{aligned}
{\left[\bar{G}_{k, R}^{T}+j \bar{G}_{k, I}^{T}\right]=} & {\left[\bar{G}_{k-1, R}^{T}+j \bar{G}_{k-1, I}^{T}\right] } \\
& -\frac{\mu}{\bar{r}_{k}^{T} \bar{r}_{k}}\left[\left(\tanh \left(\omega\left(\Phi_{R}+1\right)\right)+\tanh \left(\omega\left(\Phi_{R}-1\right)\right)\right) \bar{R}_{k, R}^{T}\right. \\
& \left.-j\left(\tanh \left(\omega\left(\Phi_{R}+1\right)\right)+\tanh \left(\omega\left(\Phi_{R}-1\right)\right)\right) \bar{R}_{k, I}^{T}\right] \\
{\left[\bar{G}_{k, R}^{T}+j \bar{G}_{k, I}^{T}\right]=} & {\left[\bar{G}_{k-1, R}^{T}+j \bar{G}_{k-1, I}^{T}\right] } \\
& -\frac{\mu}{\bar{r}_{k}^{T} \bar{r}_{k}}\left[\left(\tanh \left(\omega\left(\Phi_{I}+1\right)\right)+\tanh \left(\omega\left(\Phi_{I}-1\right)\right)\right) \bar{R}_{k, I}^{T}\right. \\
& \left.-j\left(\tanh \left(\omega\left(\Phi_{I}+1\right)\right)+\tanh \left(\omega\left(\Phi_{I}-1\right)\right)\right) \bar{R}_{k, R}^{T}\right]
\end{aligned}
$$

And we add (28) and (29) which yields

$$
\begin{aligned}
2\left[\bar{G}_{k, R}^{T}+j \bar{G}_{k, I}^{T}\right] & =2\left[\bar{G}_{k-1, R}^{T}+j \bar{G}_{k-1, I}^{T}\right] \\
& -\frac{\mu}{\overline{\mathrm{r}}_{\mathrm{k}}^{\mathrm{T}} \overline{\mathrm{r}}_{\mathrm{k}}}\left[\left(\tanh \left(\omega\left(\Phi_{R}+1\right)\right)+\tanh \left(\omega\left(\Phi_{R}-1\right)\right)\right) \bar{R}_{k, R}^{T}\right. \\
& -\mathrm{j}\left(\tanh \left(\omega\left(\Phi_{R}+1\right)\right)+\tanh \left(\omega\left(\Phi_{R}-1\right)\right)\right) \bar{R}_{k, I}^{T} \\
& +\left(\tanh \left(\omega\left(\Phi_{I}+1\right)\right)+\tanh \left(\omega\left(\Phi_{I}-1\right)\right)\right) \bar{R}_{k, I}^{T} \\
& \left.-\mathrm{j}\left(\tanh \left(\omega\left(\Phi_{I}+1\right)\right)+\tanh \left(\omega\left(\Phi_{I}-1\right)\right)\right) \bar{R}_{k, R}^{T}\right]
\end{aligned}
$$

We divide (30) by 2 and define $E_{k}$ as a complex error indicator

$$
\mathfrak{R}\left\{E_{k}\right\}=\frac{\tanh \left(\omega\left(\Phi_{R}+1\right)\right)+\tanh \left(\omega\left(\Phi_{R}-1\right)\right)}{2}
$$




$$
\widetilde{J}\left\{E_{k}\right\}=\frac{\tanh \left(\omega\left(\Phi_{I}+1\right)\right)+\tanh \left(\omega\left(\Phi_{I}-1\right)\right)}{2}
$$

We can obtain

$$
\left[\bar{G}_{k, R}^{T}+j \bar{G}_{k, I}^{T}\right]=\left[\bar{G}_{k-1, R}^{T}+j \bar{G}_{k-1, I}^{T}\right]-\frac{\mu}{\bar{r}_{k}^{T} \bar{r}_{k}}\left(\Re\left\{E_{k}\right\}+j \Im\left\{E_{k}\right\}\right)\left(\bar{R}_{k, R}^{T}-j \bar{R}_{k, I}^{T}\right)
$$

Simplifying (33), we can obtain the following complex type of NAMSER algorithm,

$$
\bar{G}_{k}=\bar{G}_{k-1}-\mu E_{k} \frac{\bar{R}_{k}^{*}}{\bar{R}_{k}^{H} \bar{R}_{k}+\sigma}
$$

Where $\mu$ is step siz and $\sigma$ is a constant. For BPSK sources, we have

$$
E_{k}=\left\{\begin{array}{l}
\frac{\tanh \left(\omega\left(\bar{G}_{k-1}^{T} \bar{R}_{k}-s_{k}+1\right)\right)-1}{2}, \text { if } s_{k}=1 \\
\frac{\tanh \left(\omega\left(\bar{G}_{k-1}^{T} \bar{R}_{k}-s_{k}-1\right)\right)+1}{2}, \text { if } s_{k}=-1
\end{array}\right.
$$

In the case of multi-level modulation like $M$-ary QAM, we can extend the equalizer algorithm to have the same formulation with the other two decision range $\bar{g}_{k}^{T} \bar{r}_{k}-\mathfrak{R}\left\{s_{k}\right\}>-1$ for $\sqrt{M}-1$ and $\bar{g}_{k}^{T} \bar{r}_{k}-\mathfrak{R}\left\{s_{k}\right\}<1$ for $-\sqrt{M}+1$ by

$$
\begin{aligned}
& \mathfrak{R}\left\{E_{k}\right\}=\left\{\begin{array}{l}
\frac{\tanh \left(\omega\left(\Phi_{R}+1\right)\right)-1}{2} \text {, if } \mathfrak{R}\left\{\mathrm{s}_{\mathrm{k}}\right\}=\sqrt{M}-1 \\
\frac{\tanh \left(\omega\left(\Phi_{R}-1\right)\right)+1}{2} \text {,if } \mathfrak{R}\left\{\mathrm{s}_{\mathrm{k}}\right\}=-\sqrt{M}+1
\end{array}\right. \\
& \mathfrak{\Im}\left\{E_{k}\right\}=\left\{\begin{array}{l}
\frac{\tanh \left(\omega\left(\Phi_{I}+1\right)\right)-1}{2}, \text { if } \mathfrak{\Im}\left\{s_{k}\right\}=\sqrt{M}-1 \\
\frac{\tanh \left(\omega\left(\Phi_{I}-1\right)\right)+1}{2}, \text { if } \mathfrak{J}\left\{s_{k}\right\}=-\sqrt{M}+1
\end{array}\right.
\end{aligned}
$$

Note that the decision ranges of $\bar{g}_{k}^{T} \bar{r}_{k}-\mathfrak{R}\left\{s_{k}\right\}<1, \bar{g}_{k}^{T} \bar{r}_{k}-\mathfrak{R}\left\{s_{k}\right\}>-1$ include the decision range of $-1<\bar{g}_{k}^{T} \bar{r}_{k}-\mathfrak{R}\left\{s_{k}\right\}<1$. The value of the error of bias between the equalizer output and the real symbol is highly likely in the range of $(-1,1)$. Now we will derive the range of maximum step size. Let the error of equalizer be defined as

$$
\mathrm{Z}(k-1)=\left\|\bar{G}_{k-1}-\dot{\bar{G}}\right\|^{2}
$$

where $\dot{\overline{\mathrm{G}}}$ is the optimum equalizer, we can obtain the mean error for the next instant as follows,

$$
\begin{array}{r}
\mathrm{E}[Z(k)]=\mathrm{E}\left[\left\|\bar{G}_{k-1}-\mu E_{k} \frac{\bar{R}_{k}^{*}}{\bar{R}_{k}^{H} \bar{R}_{k}}-\dot{\bar{G}}\right\|^{2}\right]=\mathrm{E}\left[\left\|\bar{G}_{k-1}-\dot{\bar{G}}-\mu E_{k} \frac{\bar{R}_{k}^{*}}{\bar{R}_{k}^{H} \bar{R}_{k}}\right\|^{2}\right] \\
=\mathrm{E}[Z(k-1)]+\mathrm{E}\left[\left\|\frac{\mu^{2}\left\|E_{k}\right\|^{2}}{\bar{R}_{k}^{H} \bar{R}_{k}}\right\|^{2}\right]-\mathrm{E}\left[\left\|\frac{\mu\left(E_{k}^{*} \Phi+E_{k} \Phi^{*}\right)}{\bar{R}_{k}^{H} \bar{R}_{k}}\right\|^{2}\right]
\end{array}
$$

where $\Phi=\bar{G}_{k-1}^{T} \bar{R}_{\mathrm{k}}-\dot{\bar{G}}_{k-1}^{T} \bar{R}_{k}$. To converge, we force $\mathrm{E}[Z(k)]-\mathrm{E}[Z(k-1)] \leq 0$, which yields

Hence, we obtain

$$
\mu^{2} E\left[\left\|E_{k}\right\|^{2}\right]-\mu E\left[\left(E_{k}^{*} \Phi+E_{k} \Phi^{*}\right)\right] \leq 0
$$




$$
\begin{gathered}
\mu \leq \frac{E\left[E_{k}^{*} \Phi+E_{k} \Phi^{*}\right]^{2}}{E\left[\left\|E_{k}\right\|^{2}\right]}=\frac{2 E\left[\mathfrak{R}\left\{E_{k}^{*} \Phi^{*}\right\}\right]}{E\left[\left\|E_{k}\right\|^{2}\right]} \\
=\frac{2 E\left[\mathfrak{R}\left\{E_{k}\right\} \mathfrak{R}\{\Phi\}+\Im\left\{E_{k}\right\} \mathfrak{J}\{\Phi\}\right]}{E\left[\mathfrak{R}\left\{E_{k}\right\}^{2}+\mathfrak{J}\left\{E_{k}\right\}^{2}\right]} \\
\mu \leq \frac{E\left[\left(E_{k}^{*} \Phi+E_{k} \Phi^{*}\right)\right]}{E\left[\left\|E_{k}\right\|^{2}\right]}=\frac{2 E\left[\Re\left(E_{k}^{*} \Phi\right)\right]}{E\left[\left\|E_{k}\right\|^{2}\right]}=\frac{2 E\left[\Re\left\{E_{k}\right\} \Re\{\Phi\}+\Im\left\{E_{k}\right\} \Im\{\Phi\}\right]}{E\left[\Re\left\{E_{k}\right\}^{2}+\mathfrak{J}\left\{E_{k}\right\}^{2}\right]}
\end{gathered}
$$

By the assumptions of $\mathrm{E}\left[\Re\left\{E_{k}\right\}\right]=\mathrm{E}\left[\Im\left\{E_{k}\right\}\right]$ and $\mathrm{E}\left[\Re\left\{E_{k}\right\}^{2}\right]=\mathrm{E}\left[\Im\left\{E_{k}\right\}^{2}\right]$, we can achieve

$$
0<\mu \leq \frac{2 \mathrm{E}\left[\Re\left\{E_{k}\right\} \Re\{\Phi\}\right]}{\mathrm{E}\left[\Re\left\{E_{k}\right\}^{2}\right]}
$$

Then we define the optimal step size which refers to the NLMS algorithm [23] as follows,

\subsection{Turbo Equalization}

$$
\mu_{\text {optimal }}=\frac{E\left[\Re\left\{E_{k}\right\} \Re\{\Phi\}\right]}{\mathrm{E}\left[\Re\left\{E_{k}\right\}^{2}\right]}
$$

In this section, we introduce how the turbo decoder operates and connects to the equalizer to execute iteration and become a turbo equalizer. In Fig. 3, we show that the content of turbo decoder which we follow the method of [24]. The turbo encoder involves two RSC encoder, so the turbo decoder also has two sub-decoder for each encoder. In encoder, we encode the data into three parts including systematic sequence which involve information of data, parity sequence of code 1 and parity sequence of code 2 . So the coded estimated data also involves three parts which consists data bit signal $\hat{x}_{i, k}$, parity bit signal $\hat{x}_{p 1, k}$ of code 1 and parity bit signal $\hat{x}_{p 2, k}$ of code 2 .

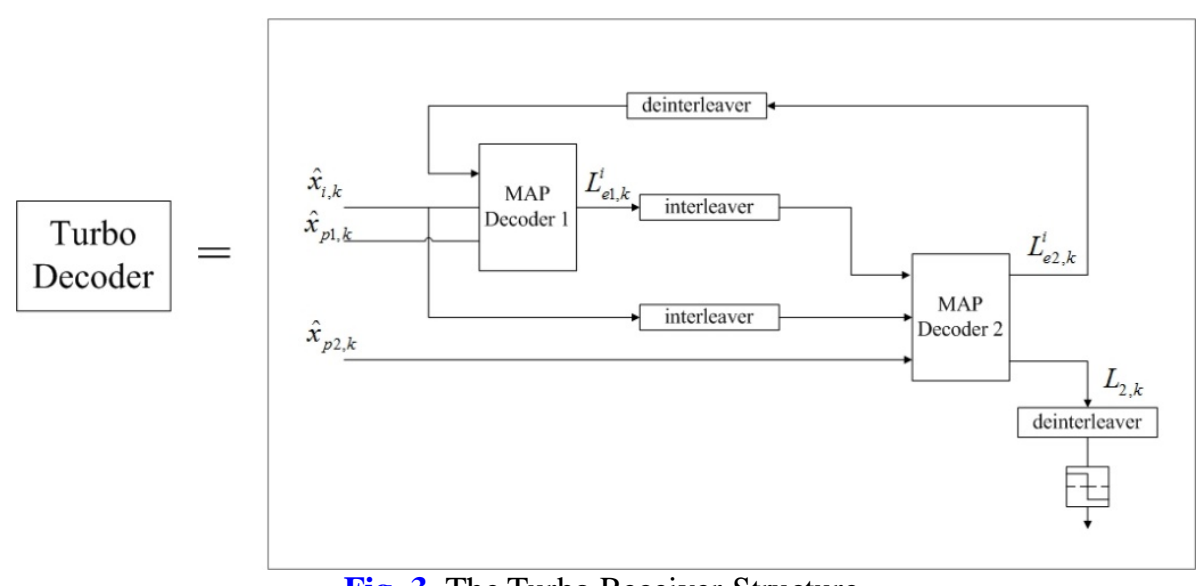

Fig. 3. The Turbo Receiver Structure.

The equalized signal sequence $\hat{s}_{k}$ is de-mapped to the estimate of the coded vector $\hat{x}_{k}$. The output of decoder 1 is the log-likelihood ratio of the transmitted bits conditioned on the equalized coded sequence. First, we use the receiver signal of code 1 to obtain

and we do the same thing in decoder 2 ,

$$
L_{1, k}=\log \frac{P\left[d_{k}=1 \mid \hat{x}_{k}\right]}{P\left[d_{k}=0 \mid \hat{x}_{k}\right]}
$$

$$
L_{2, k}=\log \frac{P\left[d_{k}=1 \mid \hat{x}_{k}\right]}{P\left[d_{k}=0 \mid \hat{x}_{k}\right]}
$$


The extrinsic information for data and parity bits for decoder 1 are denoted as $L_{e 1, k}^{i}$ and $L_{e 1, k}^{p}$ which are calculated as follows,

$$
\begin{gathered}
L_{e 1, k}^{i}=L_{1, k}-L_{a 1, k}^{i}-L_{C} \hat{X}_{i, k} \\
L_{e 1, k}^{p}=L_{1, k}-L_{C} \hat{X}_{p 1, k}
\end{gathered}
$$

where $L_{a 1, k}^{I}$ is the a prior information of data bit, and also is the extrinsic information $L_{e 2, k}^{I}$ of information bit from the decoder 2 after de-interleaving and

$$
L_{C}=a \frac{4 E_{s}}{N_{0}}
$$

is defined as the channel reliability factor in [42]. The term $E_{s}$ is the transmitted energy per symbol, $a$ is the fading amplitude and $N_{0}$ is the noise power. For non-fading AWGN channels, $a=1$. The ratio is defined as the Signal to Noise Ratio (SNR) of the channel. The output of decoder 2 is in a similar method like decoder 1 which yields

$$
\begin{gathered}
L_{e 2, k}^{i}=L_{2, k}-L_{a 2, k}^{i}-L_{C} \hat{x}_{i, k} \\
L_{e 2, k}^{p}=L_{2, k}-L_{C} \hat{x}_{p 2, k}
\end{gathered}
$$

where $L_{a 2, k}^{i}$ is the a prior information of data bit, and also is the extrinsic information $L_{e 1, k}^{i}$ of information bit from the decoder 1 after interleaving. Then we use the data bit extrinsic information $L_{e 2, k}^{i}$ of decoder 2 and the parity bit extrinsic information $L_{e 1, k}^{p}$ or $L_{e 2, k}^{p}$ to calculate the desired information of $s_{k}$ which is soft decision detected (44) and final output detection of data $\hat{d}_{k}$ which is hard decision detected (45) using the following equation,

$$
\begin{gathered}
s_{k}^{d}=\tanh \left(L_{e 2, k}^{i}\right)+j \tanh \left(L_{e, k}^{p}\right) \\
\hat{d}_{k}=\frac{\operatorname{sgn}\left(L_{e 2, k}^{i}\right)+1}{2}
\end{gathered}
$$

where $L_{e, k}^{p}$ could be $L_{e 1, k}^{p}$ or $L_{e 2, k}^{p}$ and is chosen according to the puncture of parity bits. On the other hand, we use $L_{2, k}$ which is hard decision to do iteration as following. We substitute $s_{k}$ of $\Phi_{R}=\bar{g}_{k-1}^{T} \bar{r}_{k}-\mathfrak{R}\left\{s_{k}\right\}$ and $\Phi_{I}=\bar{g}_{k-1}^{T} \mathbf{P} \bar{r}_{k}-\mathfrak{J}\left\{s_{k}\right\}$ with the soft decision $s_{k}^{d}$ in (44). The $s_{k}^{d}$ which is an error-less data estimation will send back to NAMSER equalizer in every iteration to optimize estimation of equalizer. Because we want to obtain the original transmitted signal to send back to equalizer, so we use the soft decision to get a close value of it. If we use hard decision in this way, the interference will not be removed clearly and it will cause error propagation. So we use the soft value to correct error progressively with iteration. A more accurate estimation can help equalizer to equalize signal. As a result, they can interact and increase the performance of system.

\section{Simulation Results}

In this section, the performance, parameters of the circumstance, lookup table interval and iteration times are discussed. The performance of turbo equalization with the following algorithms, normalized adaptive minimal symbol error rate (NAMSER), adaptive minimal symbol error rate (AMSER), minimum mean square error (MMSE) and least mean square (LMS) in MMIMO SC-FDMA system will be verified by Monte Carlo simulation method. The channel model is the spatial channel model (SCM) proposed in [40]. We simulate our proposed system in the QPSK modulation and channel model are rural area with 2 path taps, suburban with 6 path taps and urban with 11 path taps environment. There are one or two 
antennas for uplink transmission, and three or two hundred fifty six antennas at receiver. The parameter of NAMSER equalizer $\omega$ is defaulted as 1000 which control hyperbolic tangent, the normalized factor $\sigma$ is defaulted as 0.3 and step size $\mu$ is 0.65 . The FFT sizes are $N=256$ and $M$ $=8$. The encoder used is the recursive convolutional codes (RSC). The code rate is $1 / 2$ under three different channel encoders $(2,1,3),(2,1,4)$ and $(2,1,5)$ where the last entry denotes the memory order $m$. The random interleaver is employed. The BCJR algorithm is used in turbo decoder.

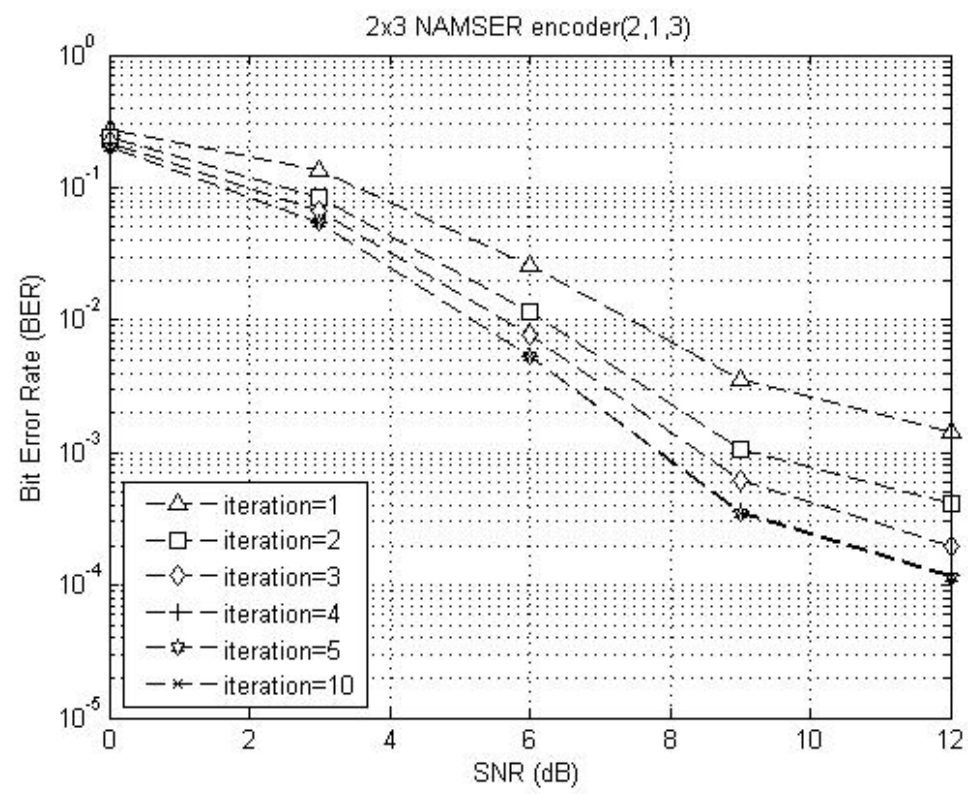

Fig. 4. The BER performance of the proposed scheme in suburban (encode $(2,1,3)$ in 2x3 antenna).

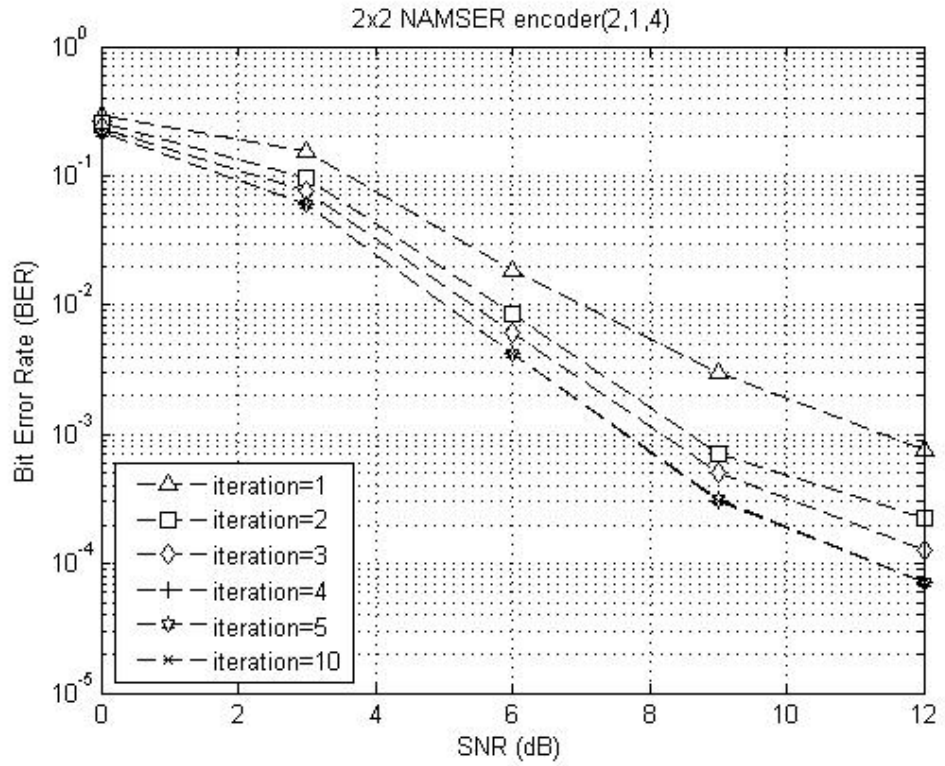

Fig. 5. The BER performance of the proposed scheme in suburban (encode $(2,1,4)$ in $2 \times 2$ antenna). 
Fig. 4 shows the bit error rate performance of every iteration of our scheme in $2 x 3$ antennas and turbo encoder is $(2,1,3)$.We can see that the performances of the fourth iteration, the fifth iteration and the tenth iteration are almost the same. At the fifth iteration, the proposed scheme converges. Fig. 5 shows the bit error rate performance of every iteration of our scheme in $2 \times 2$ antennas and turbo encoder is $(2,1,4)$. We can see that the performances of the fifth and tenth iteration are almost the same, so we know that our scheme converges at the fifth iteration. Fig. 6 shows the bit error rate performance of every iteration of our scheme in 2x3 antennas and turbo encoder is $(2,1,4)$. We can see that the performances of the fifth and tenth iteration are almost the same. At the fifth iteration, the proposed scheme converges. If we compare the figures 5 and 6, some BER improvement can be achieved if 2x3 antenna is employed (compared with that of $2 \times 2$ antenna). Fig. 7 shows the bit error rate performance of every iteration of our scheme in $2 \times 2$ antenna and turbo encoder is $(2,1,5)$. We can see that the performances of the fifth and tenth iteration are almost the same. At the fifth iteration, the proposed scheme converges. Fig. 8 shows the bit error rate performance of every iteration of our scheme in $2 \times 3$ antennas and turbo encoder is $(2,1,5)$. We can see that the performances of the fifth and tenth iteration are almost the same. At the fifth iteration, the proposed scheme converges. From the simulations of Fig. 7 and $\mathbf{8}$, we find that if we employ more powerful error correction code, the BER performance of $2 \times 3$ antenna is almost the same as that of the $2 \times 2$ antenna. Fig. 9 shows the bit error rate performances of using LUT approximation and accurate value in suburban environment under the same fifth iteration. We compare the influence in amount of LUT location. We can see the performance which the amount of LUT location is 2 and 15 and using accurate value of hyperbolic tangent are close when $\omega$ is fully large. Fig. 10 shows the bit error rate performance of using different $\omega$ in urban environment under the same fifth iteration. We discuss what value of $\omega$ is just fully large. We can see that the performance are close when $\omega$ is bigger than 100 . So we define that $\omega=100$ is fully large. Fig. 11 shows the bit error rate performance of different value of normalized factor $\sigma$ in suburban environment under the same fifth iteration. We compare the values $\sigma$ is $0.1,1$ and 10 . We can see the performance of range 0.1 to 10 are almost the same and $\sigma=0.3$ is the best. But $\sigma=10$ becomes worse. We can choose $\sigma$ in range of 0.1 to 1 . Fig. 12 shows the bit error rate performance of fifth iteration of our scheme in 1x256 antenna configuration and turbo encoder is $(2,1,5)$ in rural area at fifth iteration. We find that the performance of the proposed NAMSER algorithm is well better than existing AMSER, MMSE and LMS algorithms. Fig. 13 shows the bit error rate performance of fifth iteration of our scheme in 2x256 antenna configuation and turbo encoder is $(2,1,5)$ in rural area at fifth iteration. We find that the performance of the proposed NAMSER algorithm is well better than existing AMSER, MMSE and LMS algorithms. Fig. 14 shows the bit error rate performance of fifth iteration of our scheme in 1x256 antenna cinfiguation and turbo encoder is $(2,1,5)$ in suburban at fifth iteration. We find that the performance of the proposed NAMSER algorithm is well better than existing AMSER, MMSE and LMS algorithms. Fig. 15 shows the bit error rate performance of fifth iteration of our scheme in 2x256 antenna configuation and turbo encoder is $(2,1,5)$ in suburban at fifth iteration. We find that the performance of the proposed NAMSER algorithm is well better than existing AMSER, MMSE and LMS algorithms. If we compare the figures 14 and 15, some BER improvement can be achieved if 2x256 antenna is employed (compared with that of 1x256 antenna). Fig. 16 shows the bit error rate performance of fifth iteration of our scheme in 1x256 antenna configuation and turbo encoder is $(2,1,5)$ in urban at fifth iteration. We find that the performance of the proposed NAMSER algorithm is well better than existing AMSER, MMSE and LMS algorithms. 


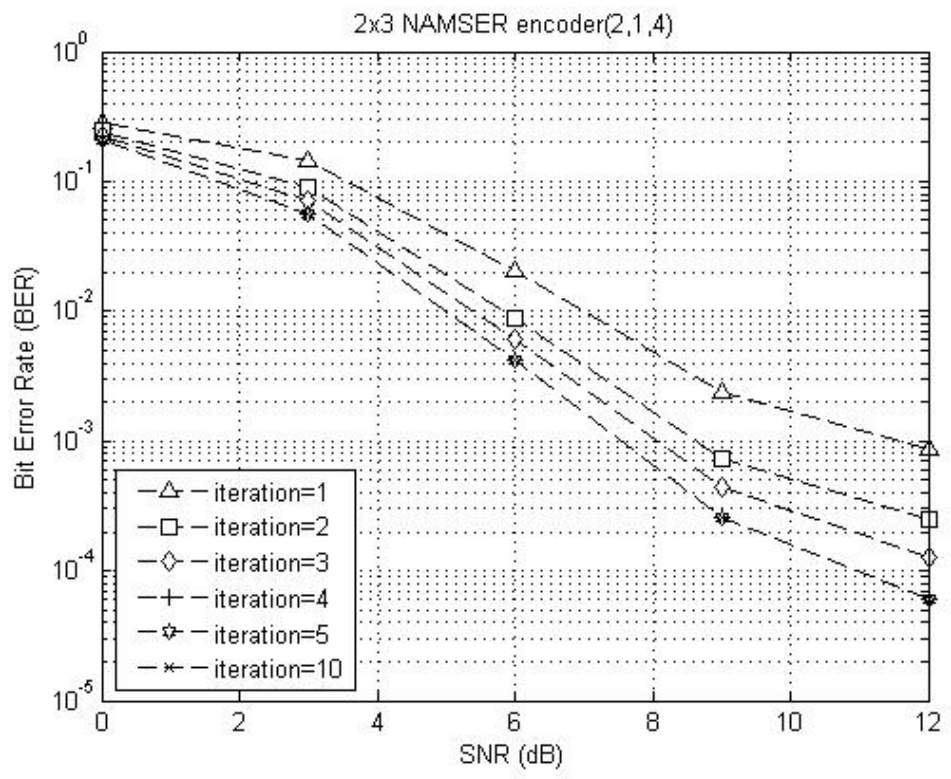

Fig. 6. The BER performance of the proposed scheme in suburban (encode $(2,1,4)$ in 2x3 antenna).

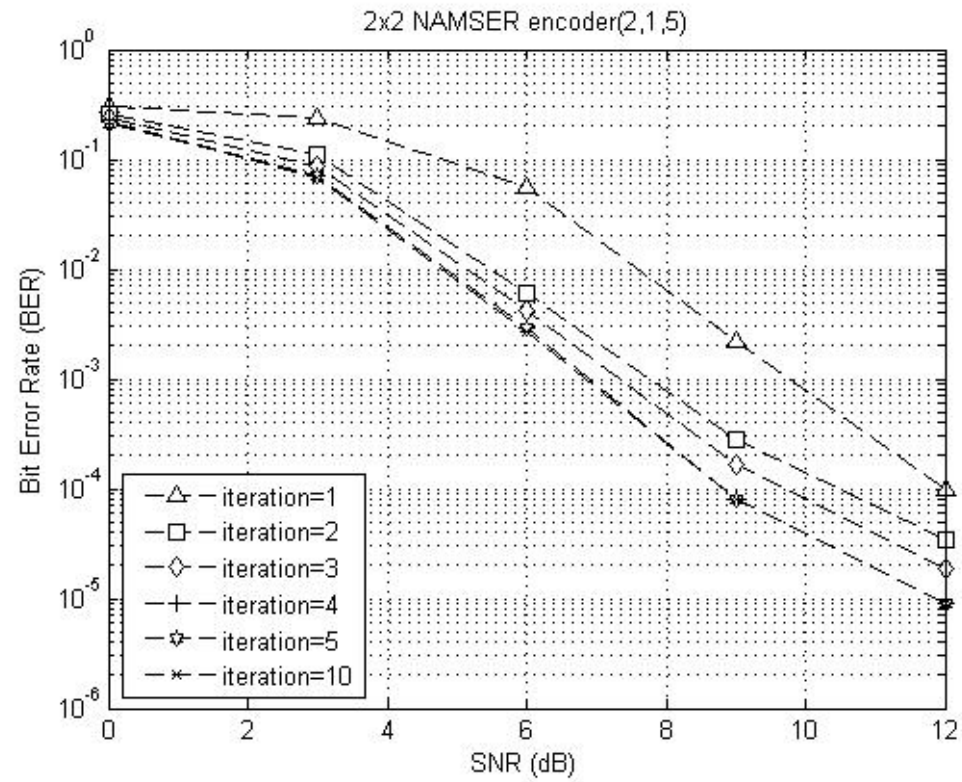

Fig. 7. The BER performance of the proposed scheme in suburban (encode $(2,1,5)$ in 2x2 antenna). 


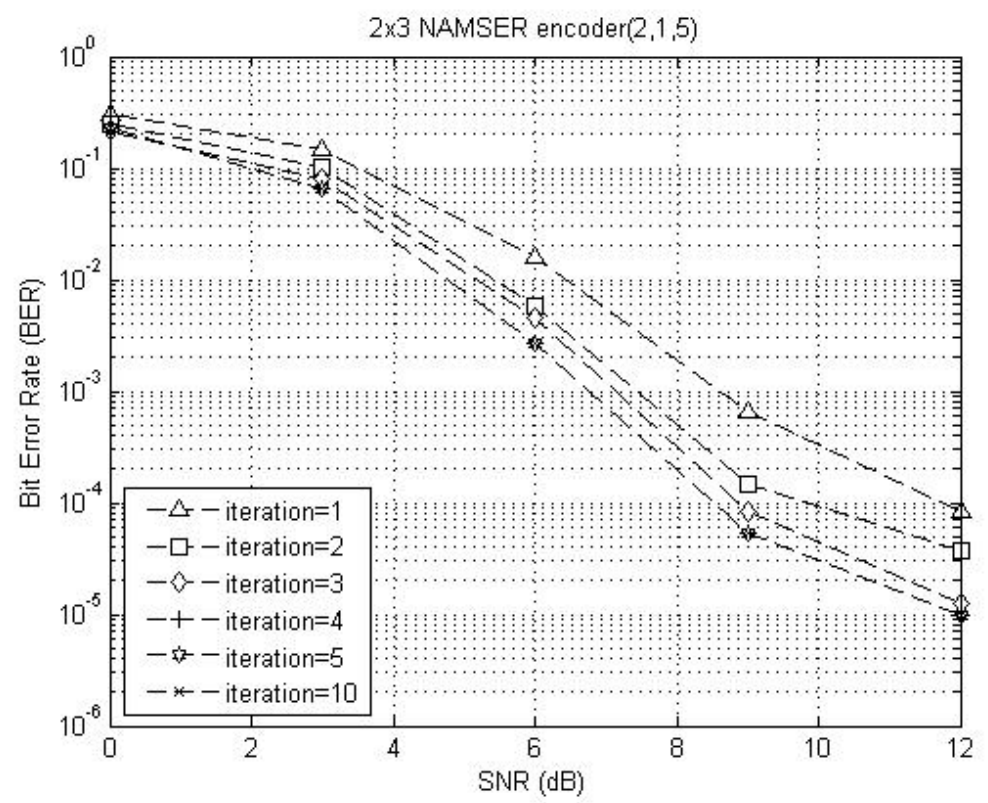

Fig. 8. The BER performance of the proposed scheme in suburban (encode $(2,1,5)$ in 2x3 antenna).

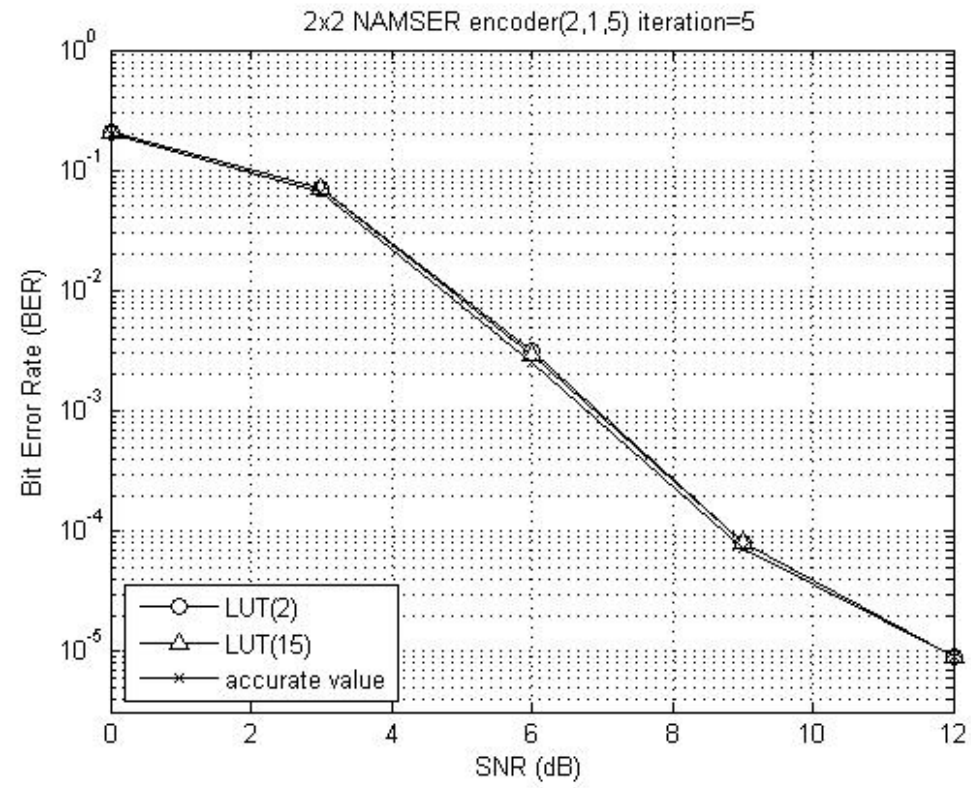

Fig. 9. The BER performance of the proposed scheme with LUT and accurate value of tanh. 


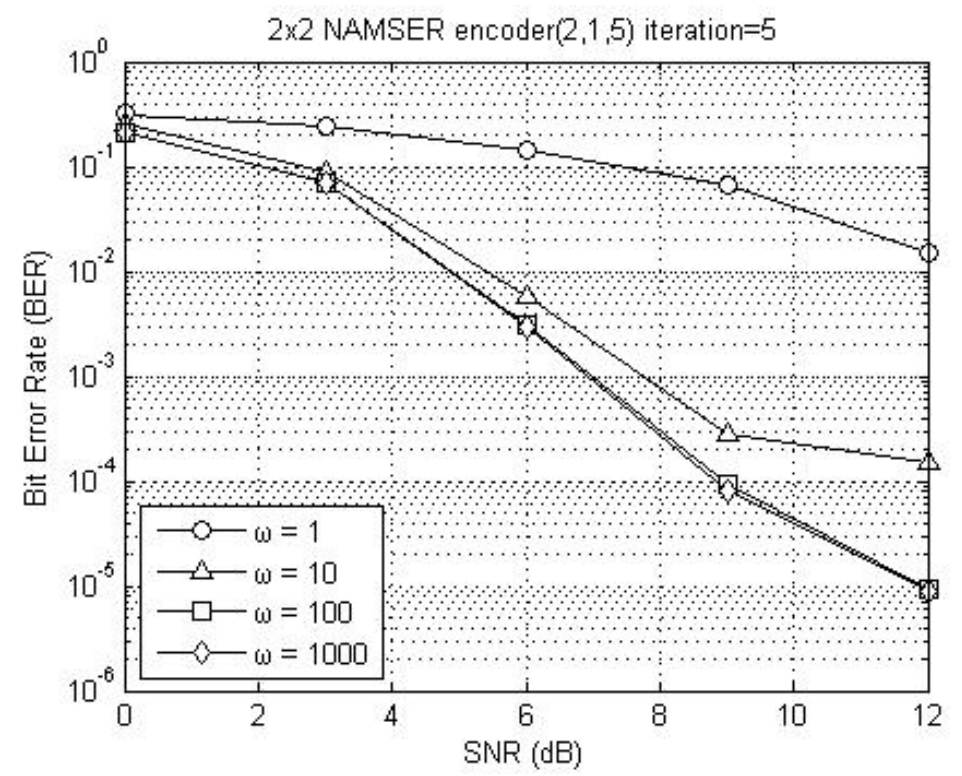

Fig. 10. The BER performance of the proposed scheme with different $\omega$.

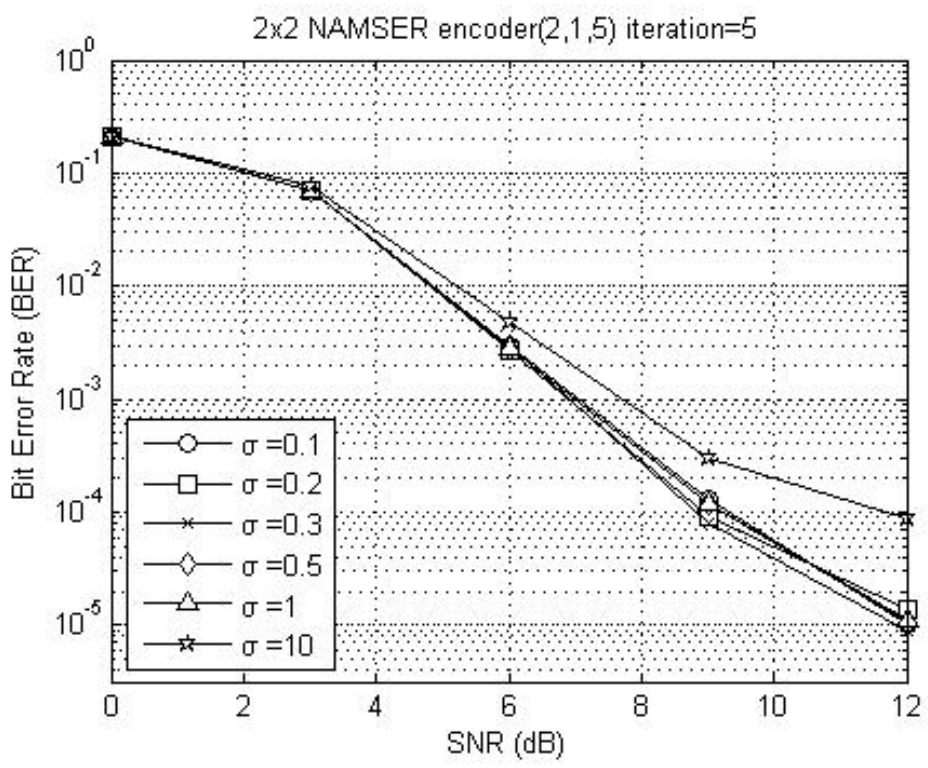

Fig. 11. The BER performance of the proposed scheme with different $\sigma$. 


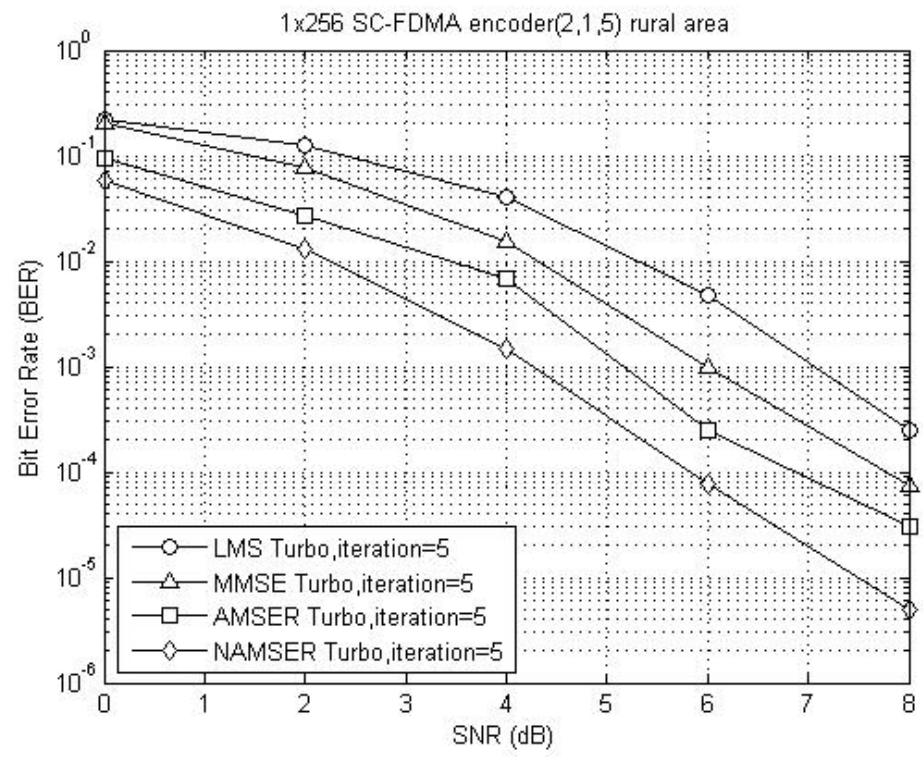

Fig. 12. The BER performance comparison of the proposed scheme (NAMSER) and three existing scheme in rural area (encode $(2,1,5)$ in 1x256 antenna).

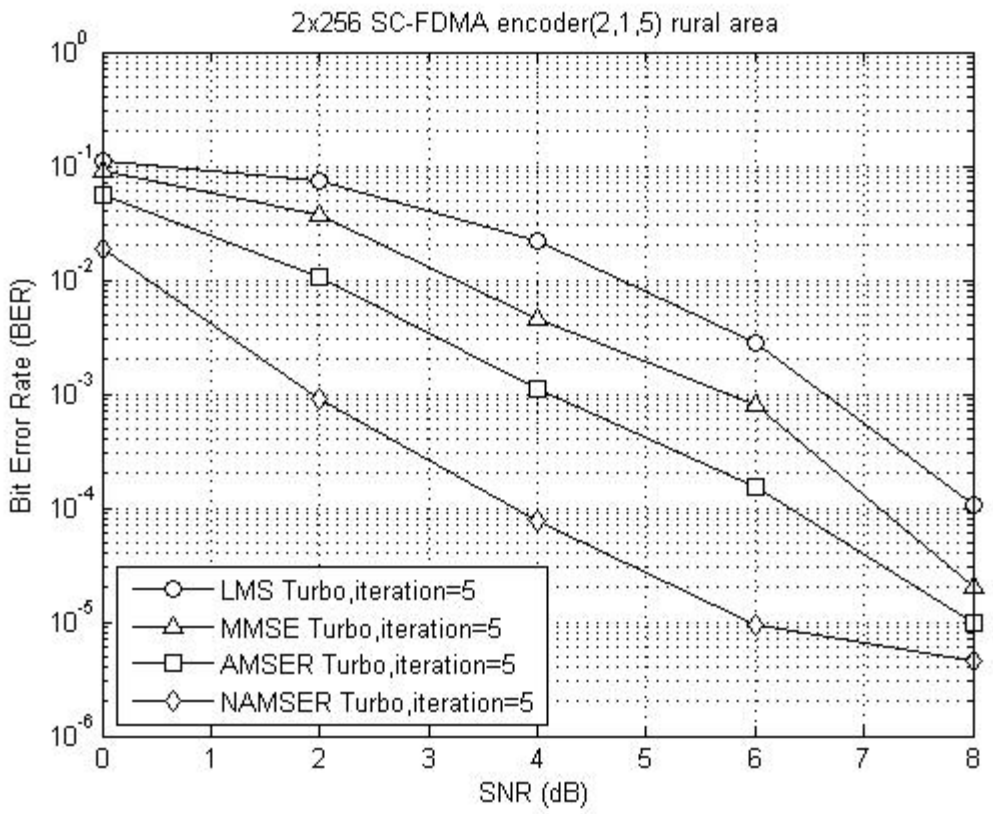

Fig. 13. The BER performance comparison of the proposed scheme (NAMSER) and three existing scheme in rural area (encode $(2,1,5)$ in 2x256 antenna). 


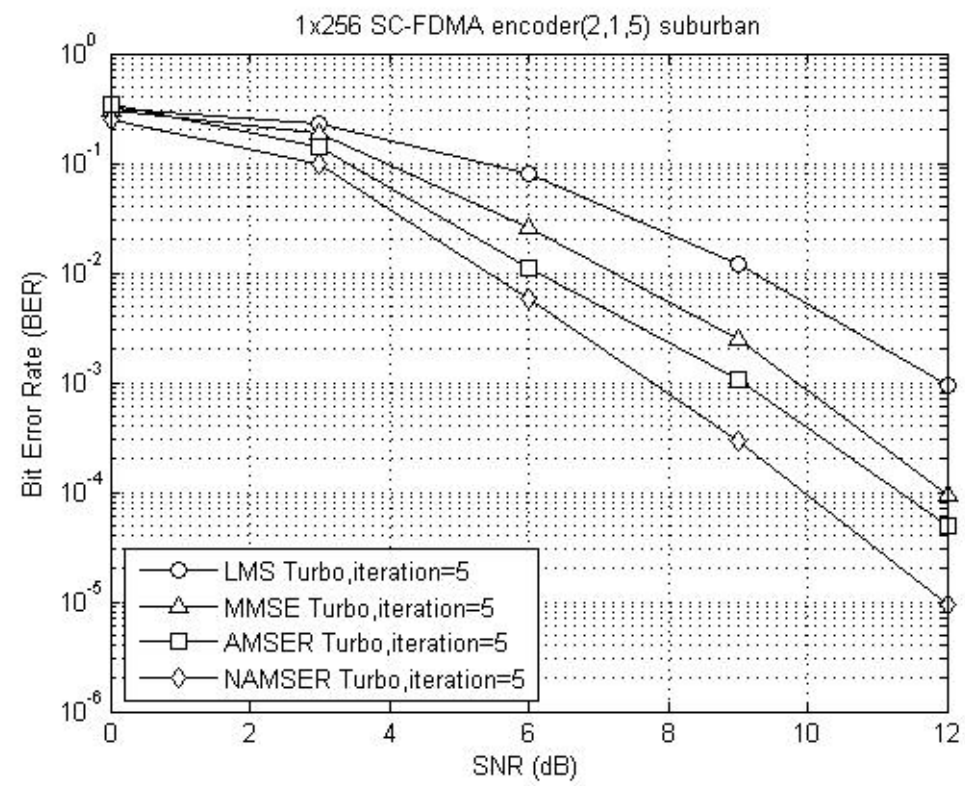

Fig. 14. The BER performance comparison of the proposed scheme (NAMSER) and three existing scheme in suburban (encode $(2,1,5)$ in 1 x256 antenna).

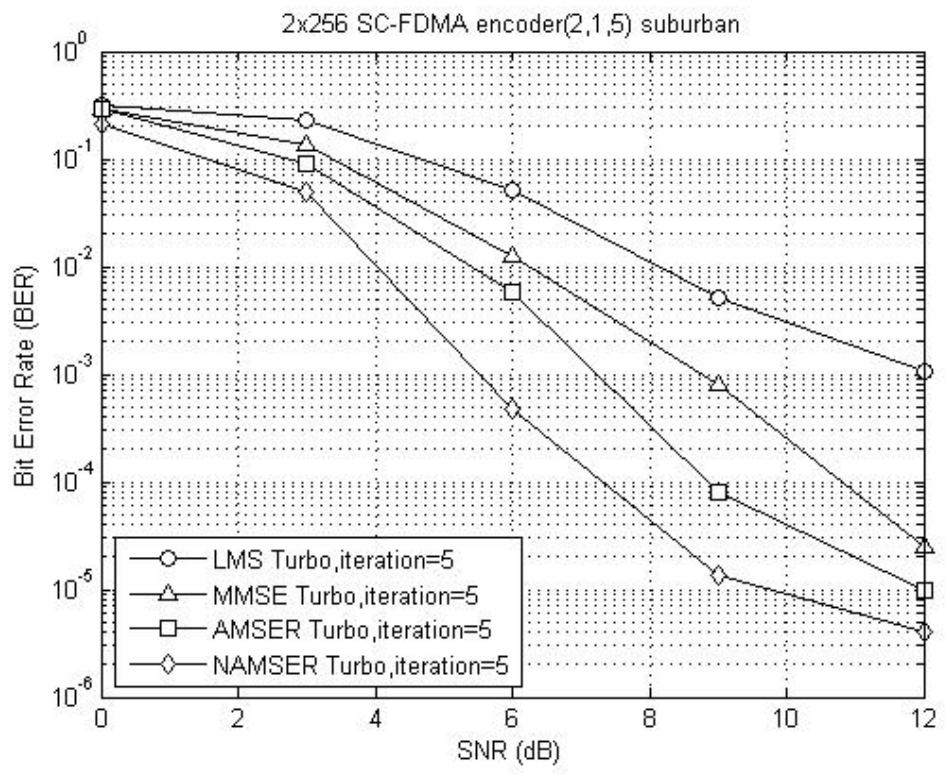

Fig. 15. The BER performance comparison of the proposed scheme (NAMSER) and three existing scheme in suburban (encode $(2,1,5)$ in 2x256 antenna). 


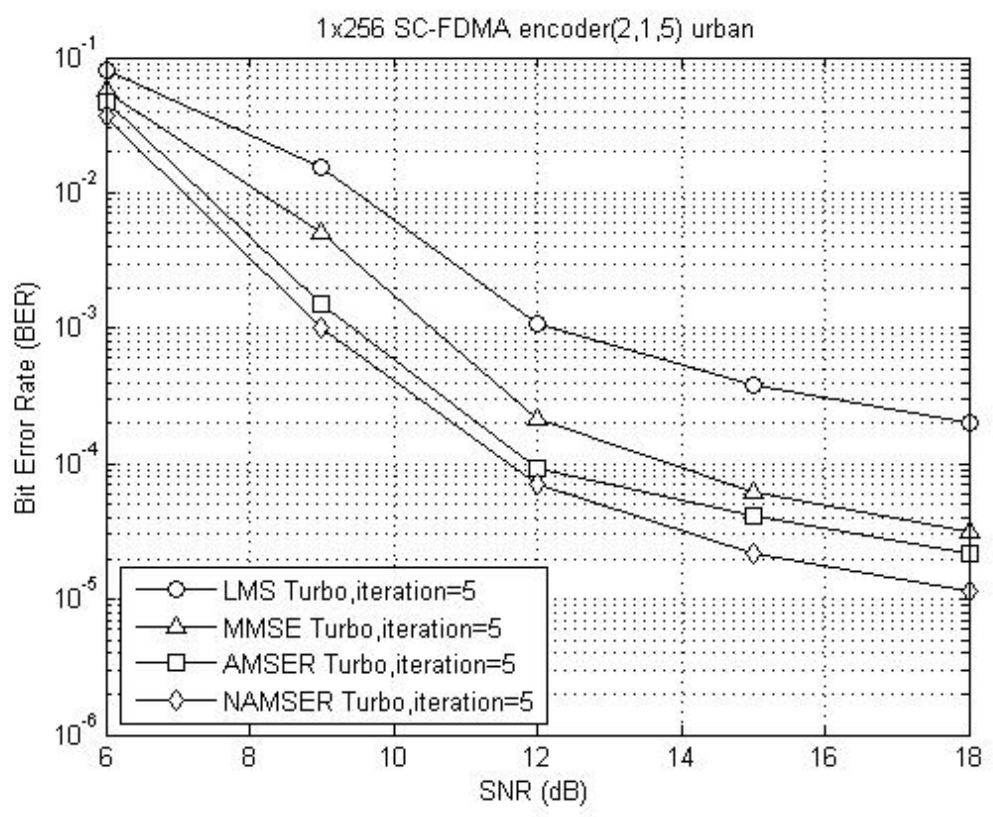

Fig. 16. The BER performance comparison of the proposed scheme (NAMSER) and three existing scheme in urban (encode $(2,1,5)$ in 1 x256 antenna).

\section{Conclusion}

This paper investigates the MMIMO SC-FDMA communication system with spatial multiplexing and proposes a low-complexity adaptive turbo NAMSER equalizer for data detection. The iterative architecture can enhance the bit-error-rate performance of the original NAMSER equalizer. The performances of the proposed turbo NAMSER scheme and the existing AMSER algorithm, turbo LMS scheme and turbo MMSE scheme are compared in different channel environments. The performance of the proposed turbo NAMSER scheme is better than that of the three existing schemes.

\section{References}

[1] H. G. Myung, J. Lim and D. J. Goodman, "Single carrier FDMA for uplink wireless transmission,” IEEE Vehicular Technology Magazine, vol. 1, no. 3, pp. 30-38, Sept. 2006.

Article (CrossRef Link)

[2] H. G. Myung and D. J. Goodman, "Single Carrier FDMA: A New Air Interface for Long Term Evolution,” John Wiley \& Sons, Ltd., Oct. 2008. Article (CrossRef Link)

[3] P. Li and R. C. de Lamare, "Adaptive Decision-Feedback Detection With Constellation Constraints for MIMO Systems," IEEE Transactions on Vehicular Technology, vol. 61, no. 2, pp. 853-859, Feb. 2012. Article (CrossRef Link)

[4] M. Tüchler, A. C. Singer and R. Koetter, "Minimum Mean Squared Error Equalization Using A Priori Information," IEEE Transactions on Signal Processing, vol. 50, no. 3, pp. 673-683, Mar. 2002. Article (CrossRef Link)

[5] R. Koetter, A. C. Singer and M. Tüchler, "Turbo equalization,” IEEE Signal Processing Magazine, vol. 21, no. 1, pp. 67-80, Jan. 2004. Article (CrossRef Link) 
[6] M. Marey, O. A. Dobre and R. Inkol, "Blind STBC Identification for Multiple-Antenna OFDM Systems,” IEEE Transactions on Communications, vol. 62, no. 5, pp. 1554-1567, April 2014. Article (CrossRef Link)

[7] Y. Ohwatari, N. Miki, Y. Sagae, and Y. Okumura, "Investigation on Interference Rejection Combining Receiver for Space-Frequency Block Code Transmit Diversity in LTE-Advanced Downlink,” IEEE Transactions on Vehicular Technology, vol. 63, no. 1, pp. 191-203, Jan. 2014. Article (CrossRef Link)

[8] M. Ahn, C. Song and I. Lee, "Diversity Analysis of Coded Spatial Multiplexing MIMO AF Relaying Systems,” IEEE Transactions on Vehicular Technology, vol. 63, no. 7, pp. 3435-3439, Sep. 2014. Article (CrossRef Link)

[9] S. Chen, S. Tana, L. Xua, and L. Hanzoa, “Adaptive minimum error-rate filtering design: a review,” Signal Process., vol. 88, no. 7, pp. 1671-1697, July 2008. Article (CrossRef Link)

[10] M. R. McKay, I. B. Collings and A. M. Tulino, "Achievable sum rate of MIMO MMSE receivers: a general analytic framework," IEEE Transactions on Information Theory, vol. 56, no. 1, pp. 396-410, Jan. 2010. Article (CrossRef Link)

[11] C.-C. Yeh and J. R. Barry, "Adaptive minimum symbol-error rate equalization for quadrature-amplitude modulation,” IEEE Trans. Signal Process, vol. 51, no. 12, pp. 3263-3269, Dec. 2003. Article (CrossRef Link)

[12] C.-C. Yeh and J. R. Barry, "Adaptive minimum bit-error rate equalization for binary signaling," IEEE Trans. Commun., vol. 48, pp. 1226-1236, July 2000. Article (CrossRef Link)

[13] B. Mulgrew and S. Chen, "Adaptive minimum-BER decision feedback equalisers for binary signalling," Submitted to elsevier signal processing, pp. 1-17, Oct. 1999 Article (CrossRef Link)

[14] S. Chen, L. Hanzo, and B. Mulgrew, “Adaptive minimum symbol error-rate decision feedback equalization for multi-level pulse-amplitude modulation,” IEEE Trans. Signal Process., vol. 52, no. 7, pp. 2092-2101, July 2004. Article (CrossRef Link)

[15] S. Chen, L. Hanzo, and A. Livingstone, "MBER space-time decision feedback equalization assisted multiuser detection for multiple antenna aided SDMA systems," IEEE Trans. Signal Process., vol. 54, no. 8, pp. 3090-3098, Aug. 2006. Article (CrossRef Link)

[16] S. Chen, N. N. Ahmad, and L. Hanzo, “Adaptive minimum bit-error rate beamforming," IEEE Trans. Wireless Commun., vol. 4, no. 2, pp. 341-348, Feb. 2005. Article (CrossRef Link)

[17] J. Gunther and T. Moon, "Minimum symbol error rate carrier phase recovery of QPSK,” IEEE Trans. Signal Process., vol. 57, no. 8, pp. 3101-3107, Aug. 2009. Article (CrossRef Link)

[18] N. B. Jayaraj, "Minimum symbol error rate timing recovery system," thesis, Utah State University. 2010. Article (CrossRef Link)

[19] A. Hjøungnes and P. S. R. Diniz, "Minimum BER prefilter transform for communications systems with binary signaling and known FIR MIMO channel,” IEEE Signal Process. Letters, vol. 3, no. 12, pp. 234-237, Dec. 2005. Article (CrossRef Link)

[20] H.-R. Zhuang, L. Dai, S.-D. Zhou, and Y. Yao, "Low complexity per antenna rate and power control approach for closed-loop V-BLAST,” IEEE Trans. Commun., vol. 51, no. 11, pp. 1783-1787, Nov. 2003. Article (CrossRef Link)

[21] N. Wang and S.D. Blostein, "Comparison of CP-based single carrier and OFDM with power allocation,” IEEE Trans. Commun., vol. 53, no. 3, pp. 391-394, Mar. 2005. Article (CrossRef Link)

[22] M. Gong, F. Chen, H. Yu, Z. Lu and L. Hu, "Normalized Adaptive Channel Equalizer Based on Minimal Symbol-Error-Rate,” IEEE Transactions On Communications, vol. 61, no. 4, pp. 1374-1383, April 2013. Article (CrossRef Link)

[23] S. O. Haykin, Adaptive Filter Theory, Prentice Hall, 5 edition, June 2013. Article (CrossRef Link)

[24] H.-F. Wang, Y.-S. Shen, J.-C. Chang, F.-B. Ueng and K.-H. Wu, “Turbo OFDM-CDMA Receiver with Varying Stepsize in Multipath Fading Channels,” in Proc. of IEEE Global Conference on Consumer Electronics, pp. 311-314, Oct. 2013. Article (CrossRef Link)

[25] S. Dixit, and D. Nagaria, "Neural Network Implementation of Least-Mean Square Adaptive Noise Cancellation," in Proc. of International Conference on Issues and Challenges in Intelligent Computing Techniques, pp. 134-139, Feb. 2014. Article (CrossRef Link) 
[26] K. Kim, N. Kalantarova, S. S. Kozat and A. C. Singer, "Linear MMSE-Optimal Turbo Equalization Using Context Trees,” IEEE Transactions On Signal Processing, vol. 61, no. 12, pp. 3041-3055, Jun. 2013. Article (CrossRef Link)

[27] S. Saranya and B. Elango, "Implementation of PWL and LUT based Approximation for Hyperbolic Tangent Activation Function in VLSI," in Proc. International Conference on Communication and Signal Processing, pp. 1778-1782, April 2014. Article (CrossRef Link)

[28] P. K. Meher, "An optimized lookup-table for the evaluation of sigmoid function for artificial neural networks,” in Proc. IEEE VLSI System on Chip Conference, pp. 91-95, Sept. 2010 Article (CrossRef Link)

[29] E. Larsson, O. Edfors, F. Tufvesson and T. Marzetta, "Massive MIMO for next generation wireless systems,” IEEE Communications Magazine, vol. 52, no. 2, pp. 186-195, Feb. 2014 Article (CrossRef Link)

[30] M. Wu, B. Yin, G. Wang, C. Dick, J. R. Cavallaro and C. Studer, "Large-Scale MIMO Detection for 3GPP LTE: Algorithms and FPGA Implementations,” IEEE Selected Topics in Signal Processing, vol. 8, no. 5, March 2014. Article (CrossRef Link)

[31] M. B. Uddin, M. Z. I. Sarkar, K. N. Faisal and M. M. Ali, "Performance Analysis of Nakagami-m Fading Massive MIMO Channels with Linear Receivers,” in. Proc. International Conference on Computer and Information Technology, pp. 510-513, Dec. 2014. Article (CrossRef Link)

[32] Z. Xiao and Z. Li, “Analysis of Massive MIMO Systems Downlink Precoding Performance,” in Proc. of International Conference on Communications and Networking, pp. 453-456, Aug. 2014 Article (CrossRef Link)

[33] M. Wu, C. Dick, J. R. Cavallaro and C. Studer, "Iterative detection and decoding in 3GPP LTE-based massive MIMO systems,” in Proc. of IEEE Signal Processing Conference, pp. 96-100, Sept. 2014. Article (CrossRef Link)

[34] J. Zhou, Y. Ye, J. Hu, "Biased MMSE Soft-Output Detection Based on Jacobi Method in Massive MIMO,” in Proc. of IEEE International Conference Communication Problem-Solving, pp. 442-445, Dec. 2014. Article (CrossRef Link)

[35] S. K. Mohammed, "Impact of Transceiver Power Consumption on the Energy Efficiency of Zero-Forcing Detector in Massive MIMO Systems,” IEEE Transactions on Communications, vol. 62, no. 11, pp. 3874-3890, Oct. 2014. Article (CrossRef Link)

[36] Y. Li, Y.-H. Nam and B. L. Ng, “Zero-forcing Receiver in Uplink Massive MIMO,” in Proc. IEEE Globecom Workshops, pp. 140-144, Dec. 2013. Article (CrossRef Link)

[37] Y. Sheng, Z. Tan and G. Y. Li, "Single-Carrier Modulation with ML Equalization for Large-Scale Antenna Systems over Rician Fading Channels,” in Proc. IEEE International Conference on Acoustic, Speech and Signal Processing, pp. 5759-5763, May 2014. Article (CrossRef Link)

[38] T. Chen and H. Leib, “GPU Acceleration for Fixed Complexity Sphere Decoder in Large MIMO Uplink Systems," in Proc. of IEEE Conference on Electrical and Computer Engineering, pp. 771-777, May 2015. Article (CrossRef Link)

[39] Y. Wu and J. McAllister, "FPGA-based Tabu Search for Detection in Large-Scale MIMO Systems,” in Proc. of IEEE Workshop on Signal Processing Systems, pp. 1-6, Oct. 2014

Article (CrossRef Link)

[40] Spatial Channel Model for Multiple Input Multiple Output (MIMO) Simulations A Ray Tracing Simulator Based on 3GPP TR 25.996 v. 6.1.0, Developed by: Ioannis A. Xirouchakis, Undergraduate Student, Physics Department, University of Athen. Article (CrossRef Link)

[41] H. Lou and C. Xiao, "Soft-Decision Feedback Turbo Equalization for Multilevel Modulations," IEEE Transactions on Signal Processing, vol. 59, no. 1, pp. 186-195, Jan. 2011.

Article (CrossRef Link) 
[42] L. A. Perișoară, and R. Stoian, "The Decision Reliability of MAP, Log-MAP, Max-Log-MAP and SOVA Algorithms or Turbo Codes,” International Journal of Communications, vol. 2, no. 1, 2008. Article (CrossRef Link)

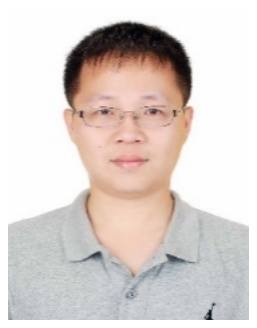

Yu-Kuan Chang was born in Kaohsiung, Taiwan, in 1986. He received the B.Sc. degree in aeronautical engineering from National Formosa University (NFU), Yunlin, Taiwan, in 2008 and the M.Sc. degree from the National Chi Nan University (NCNU), Nantou, Taiwan, in 2010. He is a member of the Communications System Laboratory, National Chung Hsing University (NCHU). His research interest is focused on MIMO MC-CDMA and LTE communication systems.

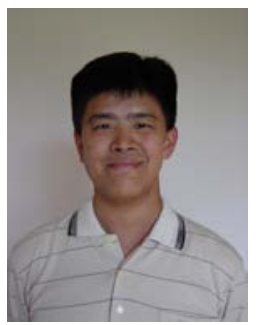

Fang-Biau Ueng received the Ph.D. degree in electronic engineering from the National Chiao Tung University, Hsinchu, Taiwan in 1995. From 1996 to 2001 he was with National Space Program Office (NSPO) of Taiwan as an associate researcher. From 2001 to 2002 he was with Siemens Telecommunication Systems Limited (STSL), Taipei, Taiwan, where he was involved in the design of mobile communication systems. Since February 2002 he has been with the department of electrical engineering, National Chung-Hsing University, Taichung, Taiwan. His areas of research interest are wireless communication and adaptive signal processing.

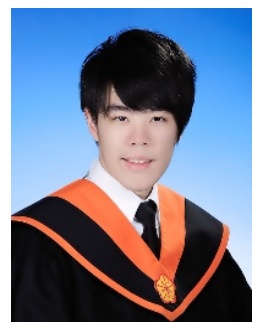

Bo-Yi Tsai was born in Tainan,, Taiwan, on November 1990. He received the B.S. degree from the Department of Optoelectronics and Communication Engineering, National Kaohsiung Normal University (NKNU), Kaohsiung, Taiwan, in 2013, and M.S. degree in Communication engineering from the National Chung Hsing University (NCHU), Taichung, Taiwan, in 2015. He was a member of the Electronic Communication Systems Laboratory, NCHU. He is currently fulfilling his mandatory military service. His research interests include Long Term Evolution (LTE), multiple antenna communications, and adaptive signal processing. 